Acta Crystallographica Section E

Structure Reports

Online

ISSN 1600-5368

\title{
N-tert-Butyl-2-methylpropanamide. Corrigendum
}

\section{Kelly A. Kluge, Diana Fridlyand, Cora E. MacBeth* and Kenneth I. Hardcastle}

Department of Chemistry, Emory University, 1515 Dickey Drive, Atlanta, GA 30322, USA

Correspondence e-mail: cora.macbeth@emory.edu

Received 16 November 2011; accepted 16 November 2011

The name of one of the authors in the paper by Kluge et al. [Acta Cryst. (2011), E67, o2143] is corrected.

In the paper by Kluge et al. (2011), the name of the second author is given incorrectly. The correct name is given above.

\section{References}

Kluge, K. A., Fridlyand, D., MacBeth, C. A. \& Hardcastle, K. I. (2011). Acta Cryst. E67, o2143. 
Acta Crystallographica Section E

\section{Structure Reports}

Online

ISSN 1600-5368

\section{N-tert-Butyl-2-methylpropanamide}

\section{Kelly A. Kluge, Diana Fridyland, Cora E. MacBeth* and Kenneth I. Hardcastle}

Department of Chemistry, Emory University, 1515 Dickey Drive, Atlanta, GA 30322, USA

Correspondence e-mail: cora.macbeth@emory.edu

Received 21 June 2011; accepted 18 July 2011

Key indicators: single-crystal X-ray study; $T=173 \mathrm{~K}$; mean $\sigma(\mathrm{C}-\mathrm{C})=0.003 \AA$; $R$ factor $=0.035 ; w R$ factor $=0.106 ;$ data-to-parameter ratio $=14.7$.

The title compound, $\mathrm{C}_{8} \mathrm{H}_{17} \mathrm{NO}$, crystallizes with two independent molecules in the asymmetric unit. In the crystal, intermolecular $\mathrm{N}-\mathrm{H} \cdots \mathrm{O}$ hydrogen bonding is observed between neighboring molecules, forming continuous molecular chains along the $c$-axis direction.

\section{Related literature}

For the synthesis of the title compound, see: De Kimpe et al. (1978); Christensen et al. (1989); Yasuhara et al. (2000); Li et al. (2003). For its use as a ligand in $\mathrm{Zr}$ and Ti complexes, see: Li et al. (2003). For background to the coordination modes of carboxamides, see: Lee \& Schafer (2007).

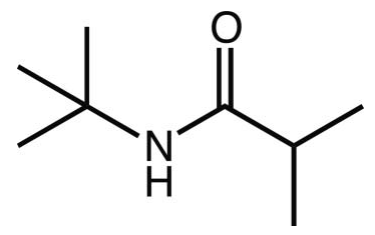

\section{Experimental}

Crystal data

$\mathrm{C}_{8} \mathrm{H}_{17} \mathrm{NO}$

$M_{r}=143.23$

Monoclinic, $P 2_{\mathrm{f}}$

$a=9.0378(6) \AA$

$b=11.3939(8) \AA$

$c=9.5390(6) \AA$

$\beta=106.133(3)^{\circ}$
Data collection

Bruker APEXII CCD

diffractometer

Absorption correction: multi-scan (SADABS; Bruker, 2008)

$T_{\text {min }}=0.858, T_{\text {max }}=0.941$

Refinement

$R\left[F^{2}>2 \sigma\left(F^{2}\right)\right]=0.035$

$w R\left(F^{2}\right)=0.106$

$S=1.00$

2662 reflections

181 parameters

1 restraint
6358 measured reflections 2662 independent reflections 2624 reflections with $I>2 \sigma(I)$ $R_{\text {int }}=0.014$

$\mathrm{H}$-atom parameters constrained $\Delta \rho_{\max }=0.19{\mathrm{e} \AA^{-3}}^{-3}$

$\Delta \rho_{\min }=-0.16 \mathrm{e}^{-3}$

Absolute structure: Flack (1983), 812 Friedel pairs

Flack parameter: $0.3(2)$

Table 1

Hydrogen-bond geometry $\left(\AA{ }^{\circ}\right)$.

\begin{tabular}{lllll}
\hline$D-\mathrm{H} \cdots A$ & $D-\mathrm{H}$ & $\mathrm{H} \cdots A$ & $D \cdots A$ & $D-\mathrm{H} \cdots A$ \\
\hline $\mathrm{N} 1-\mathrm{H} 1 A \cdots \mathrm{O} 2$ & 0.88 & 2.03 & $2.8880(16)$ & 166 \\
$\mathrm{~N} 2-\mathrm{H} 2 A \cdots \mathrm{O} 1^{\mathrm{i}}$ & 0.88 & 2.10 & $2.9735(16)$ & 169 \\
\hline
\end{tabular}

Symmetry code: (i) $x, y, z+1$.

Data collection: APEX2 (Bruker, 2008); cell refinement: SAINT (Bruker, 2008); data reduction: $S A I N T$; $\operatorname{program}(\mathrm{s})$ used to solve structure: SHELXS97 (Sheldrick, 2008); program(s) used to refine structure: SHELXL97 (Sheldrick, 2008); molecular graphics: SHELXTL (Sheldrick, 2008); software used to prepare material for publication: SHELXTL.

We acknowledge the Emory University Center for X-ray Crystallography for assistance with data collection.

Supplementary data and figures for this paper are available from the IUCr electronic archives (Reference: FJ2438).

\section{References}

Bruker (2008). APEX2, SADABS and SAINT. Bruker AXS Inc., Madison, Wisconsin, USA

Christensen, D. \& Jorgensen, K. A. (1989). J. Org. Chem. 54, 126-131.

De Kimpe, N., Verhe, R., De Buyck, L., Chys, J. \& Schamp, N. (1978). Org. Prep. Proc. Intl. 10, 149-156.

Flack, H. D. (1983). Acta Cryst. A39, 876-881.

Lee, A. \& Schafer, L. L. (2007). Eur. J. Inorg. Chem. pp. 2243-2255.

Li, C., Thomson, R. K., Gillon, B., Patrick, B. O. \& Schafer, L. L. (2003). Chem. Commun. pp. 2462-2463.

Sheldrick, G. M. (2008). Acta Cryst. A64, 112-122.

Yasuhara, T., Nagaoka, Y. \& Tomioka, K. (2000). J. Chem. Soc. Perkin Trans. 1, pp. 2901-2902. 


\section{supporting information}

Acta Cryst. (2011). E67, o2143 [doi:10.1107/S1600536811028947]

\section{N-tert-Butyl-2-methylpropanamide}

\section{Kelly A. Kluge, Diana Fridyland, Cora E. MacBeth and Kenneth I. Hardcastle}

\section{S1. Comment}

Carboxamides can be deprotonated to form monoanionic amidate ligands. These species can coordinate to transition metal ions through a variety of different coordination modes, including monodentate and bidentate coordination modes, and therefore are coordinatively versatile ligands (Lee \& Schafer, 2007). The ease of synthesis of carboxamides make them attractive ligands for a variety of transition metal mediated catalytic reactions, see: Li et al. (2003) and Lee \& Schafer (2007). Although the synthesis of this compound has been previously described, its solid-state structure has not been reported. The two molecules (A and B) of N-tert-butyl-2-methylpropanamide (Fig. 1) are stabilized by intermolecular $\mathrm{N}-\mathrm{H} \cdots \mathrm{O}$ hydrogen bonds (Table 1, Fig. 2).

\section{S2. Experimental}

The title molecule was synthesized using a modified literature procedure (Li et al., 2003). Under a nitrogen atmosphere, a $100 \mathrm{ml}$ round bottom flask was charged with $50 \mathrm{ml}$ of dichloromethane, $4.31 \mathrm{ml}(41.0 \mathrm{mmol})$ tert-butylamine, $8.55 \mathrm{ml}$ $(61.5 \mathrm{mmol})$ of triethylamine and a stir bar. The solution was cooled to $0{ }^{\circ} \mathrm{C}$ and $5.20 \mathrm{ml}(49.2 \mathrm{mmol})$ of isobutyryl chloride was added dropwise. The solution was slowly warmed to room temperature overnight. The resulting pink solution was extracted three times with $50 \mathrm{ml}$ of $0.10 \mathrm{M} \mathrm{HCl}$. The organic layer was dried over anhydrous magnesium sulfate, filtered, and concentrated to dryness to yield the desired product in $60 \%$ yield. X-ray quality crystals were obtained by slowly evaporating a chloroform solution of the product. The spectroscopic data (NMR, IR, and ESI-MS) match well with the reported values (Li et al., 2003).

\section{S3. Refinement}

The structures were solved using Direct Methods and difference Fourier techniques (SHELXTL, V6.12) (Sheldrick, 2008). Hydrogen atoms were added with the HFIX command. These were included in the final cycles of least squares refinement, with isotropic $U^{i j}$ 's that were determined by the riding model. All non-hydrogen atoms in the main residues were refined anisotropically, but residual solvent molecules in the unit cells were refined isotropically. Structure solution, refinement, and generation of publication materials were performed by using SHELX, V6.12 software. 
Molecule B

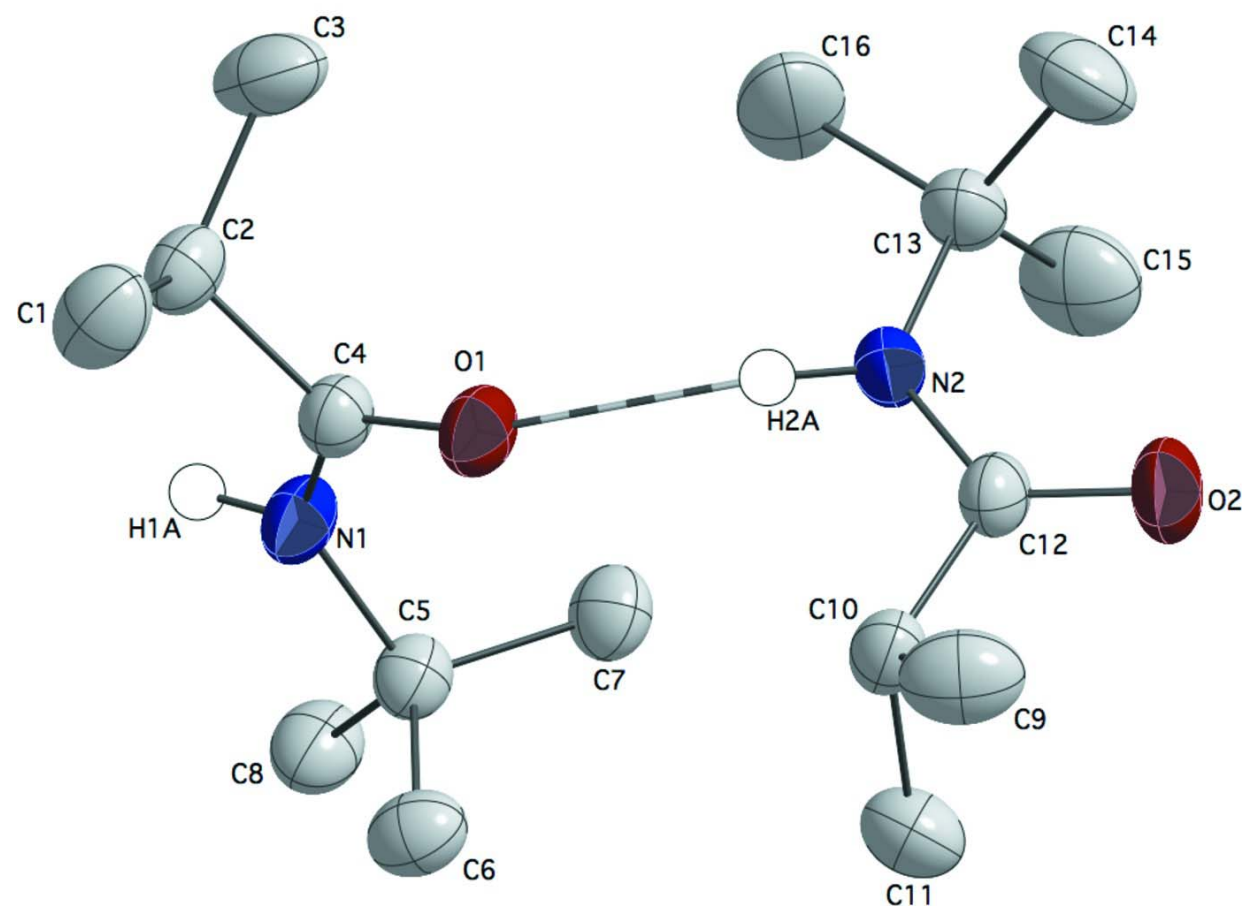

\section{Figure 1}

Molecule A and Molecule B of $\mathrm{N}$-tert-butyl-2-methylpropanamide. $\mathrm{H}$ atoms except H1A and H2A were omitted for clarity. Thermal ellipsoids are drawn at 50\% probability.

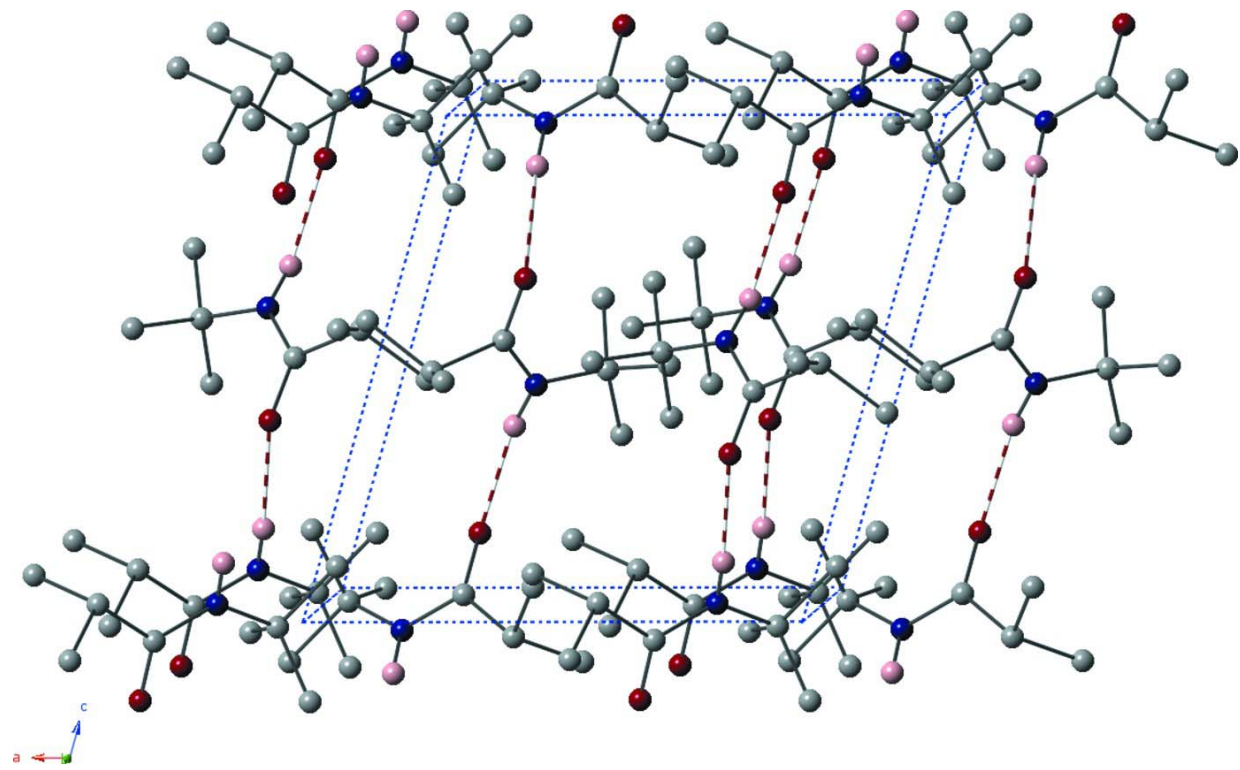

\section{Figure 2}

Molecular packing and hydrogen bonding (dashed lines) network of $N$-tert- butyl-2-methylpropanamide viewed down the $b$ axis. 


\section{N-tert-butyl-2-methylpropanamide}

Crystal data

$\mathrm{C}_{8} \mathrm{H}_{17} \mathrm{NO}$

$M_{r}=143.23$

Monoclinic, $P 2_{1}$

Hall symbol: $\mathrm{P} 2 \mathrm{yb}$

$a=9.0378(6) \AA$

$b=11.3939(8) \AA$

$c=9.5390(6) \AA$

$\beta=106.133(3)^{\circ}$

$V=943.60(11) \AA^{3}$

$Z=4$

\section{Data collection}

\section{Bruker APEXII CCD}

diffractometer

Radiation source: fine-focus sealed tube

Graphite monochromator

$\varphi$ and $\omega$ scans

Absorption correction: multi-scan

(SADABS; Bruker, 2008)

$T_{\min }=0.858, T_{\max }=0.941$

\section{Refinement}

Refinement on $F^{2}$

Least-squares matrix: full

$R\left[F^{2}>2 \sigma\left(F^{2}\right)\right]=0.035$

$w R\left(F^{2}\right)=0.106$

$S=1.00$

2662 reflections

181 parameters

1 restraint

Primary atom site location: structure-invariant direct methods

Secondary atom site location: difference Fourier map
$F(000)=320$

$D_{\mathrm{x}}=1.008 \mathrm{Mg} \mathrm{m}^{-3}$

Melting point: $393 \mathrm{~K}$

$\mathrm{Cu} K \alpha$ radiation, $\lambda=1.54178 \AA$

Cell parameters from 4549 reflections

$\theta=4.8-69.1^{\circ}$

$\mu=0.51 \mathrm{~mm}^{-1}$

$T=173 \mathrm{~K}$

Block, colourless

$0.31 \times 0.20 \times 0.12 \mathrm{~mm}$

6358 measured reflections

2662 independent reflections

2624 reflections with $I>2 \sigma(I)$

$R_{\text {int }}=0.014$

$\theta_{\text {max }}=69.1^{\circ}, \theta_{\min }=4.8^{\circ}$

$h=-10 \rightarrow 10$

$k=-13 \rightarrow 12$

$l=-11 \rightarrow 11$

Hydrogen site location: inferred from neighbouring sites

$\mathrm{H}$-atom parameters constrained

$w=1 /\left[\sigma^{2}\left(F_{0}^{2}\right)+(0.088 P)^{2}+0.0504 P\right]$

where $P=\left(F_{\mathrm{o}}{ }^{2}+2 F_{\mathrm{c}}{ }^{2}\right) / 3$

$(\Delta / \sigma)_{\max }<0.001$

$\Delta \rho_{\max }=0.19$ e $\AA^{-3}$

$\Delta \rho_{\min }=-0.16$ e $\AA^{-3}$

Absolute structure: Flack (1983), 812 Friedel pairs

Absolute structure parameter: 0.3 (2)

Special details

Geometry. All e.s.d.s (except the e.s.d. in the dihedral angle between two 1.s. planes) are estimated using the full covariance matrix. The cell e.s.d.'s are taken into account individually in the estimation of e.s.d.'s in distances, angles and torsion angles; correlations between e.s.d.'s in cell parameters are only used when they are defined by crystal symmetry. An approximate (isotropic) treatment of cell e.s.d.'s is used for estimating e.s.d.'s involving 1.s. planes.

Fractional atomic coordinates and isotropic or equivalent isotropic displacement parameters $\left(\AA^{2}\right)$

\begin{tabular}{lllll}
\hline & $x$ & $y$ & $z$ & $U_{\text {iso }} * / U_{\text {eq }}$ \\
\hline $\mathrm{N} 1$ & $0.30615(14)$ & $0.07943(12)$ & $0.55844(13)$ & $0.0364(3)$ \\
$\mathrm{H} 1 \mathrm{~A}$ & 0.2841 & 0.0677 & 0.6416 & $0.044^{*}$ \\
$\mathrm{~N} 2$ & $0.17705(14)$ & $0.08317(12)$ & $1.04274(12)$ & $0.0355(3)$ \\
$\mathrm{H} 2 \mathrm{~A}$ & 0.1857 & 0.1191 & 1.1263 & $0.043^{*}$ \\
O1 & $0.23057(13)$ & $0.17773(11)$ & $0.34325(11)$ & $0.0394(3)$ \\
O2 & $0.27727(15)$ & $0.06614(13)$ & $0.85203(12)$ & $0.0498(3)$
\end{tabular}




\begin{tabular}{|c|c|c|c|c|}
\hline $\mathrm{C} 1$ & $0.1354(2)$ & $0.35161(16)$ & $0.5354(2)$ & $0.0541(5)$ \\
\hline H1B & 0.0593 & 0.3932 & 0.5728 & $0.081^{*}$ \\
\hline $\mathrm{H} 1 \mathrm{C}$ & 0.1322 & 0.3819 & 0.4385 & $0.081^{*}$ \\
\hline H1D & 0.2385 & 0.3638 & 0.6018 & $0.081^{*}$ \\
\hline $\mathrm{C} 2$ & $0.09866(19)$ & $0.22154(14)$ & $0.52483(17)$ & $0.0383(4)$ \\
\hline $\mathrm{H} 2 \mathrm{~B}$ & 0.1043 & 0.1912 & 0.6246 & $0.046^{*}$ \\
\hline $\mathrm{C} 3$ & $-0.0618(2)$ & $0.19845(19)$ & $0.4252(3)$ & $0.0582(5)$ \\
\hline $\mathrm{H} 3 \mathrm{~A}$ & -0.1379 & 0.2398 & 0.4627 & $0.087^{*}$ \\
\hline H3B & -0.0829 & 0.1140 & 0.4220 & $0.087 *$ \\
\hline $\mathrm{H} 3 \mathrm{C}$ & -0.0680 & 0.2266 & 0.3267 & $0.087^{*}$ \\
\hline $\mathrm{C} 4$ & $0.21920(17)$ & $0.15640(14)$ & $0.46654(15)$ & $0.0342(3)$ \\
\hline $\mathrm{C} 5$ & $0.43590(17)$ & 0.01194 (14) & $0.53402(15)$ & $0.0350(3)$ \\
\hline C6 & $0.56316(19)$ & $0.09591(16)$ & $0.5211(2)$ & 0.0447 (4) \\
\hline H6A & 0.5246 & 0.1463 & 0.4354 & $0.067^{*}$ \\
\hline H6B & 0.6517 & 0.0507 & 0.5105 & $0.067^{*}$ \\
\hline $\mathrm{H} 6 \mathrm{C}$ & 0.5948 & 0.1446 & 0.6090 & $0.067^{*}$ \\
\hline $\mathrm{C} 7$ & $0.3832(2)$ & $-0.06553(16)$ & 0.39919 (17) & $0.0428(4)$ \\
\hline H7A & 0.3015 & -0.1183 & 0.4102 & $0.064^{*}$ \\
\hline H7B & 0.4704 & -0.1120 & 0.3879 & $0.064^{*}$ \\
\hline $\mathrm{H} 7 \mathrm{C}$ & 0.3437 & -0.0162 & 0.3127 & $0.064^{*}$ \\
\hline $\mathrm{C} 8$ & $0.4953(2)$ & $-0.06704(17)$ & $0.66758(18)$ & $0.0466(4)$ \\
\hline H8A & 0.4130 & -0.1203 & 0.6759 & $0.070^{*}$ \\
\hline H8B & 0.5277 & -0.0185 & 0.7556 & $0.070^{*}$ \\
\hline $\mathrm{H} 8 \mathrm{C}$ & 0.5832 & -0.1129 & 0.6568 & $0.070^{*}$ \\
\hline C9 & $0.4281(2)$ & $0.28811(19)$ & $0.9455(3)$ & $0.0580(5)$ \\
\hline H9A & 0.5106 & 0.3416 & 0.9961 & $0.087 *$ \\
\hline H9B & 0.3314 & 0.3318 & 0.9116 & $0.087^{*}$ \\
\hline H9C & 0.4542 & 0.2529 & 0.8617 & $0.087^{*}$ \\
\hline $\mathrm{C} 10$ & $0.40976(18)$ & $0.19187(15)$ & $1.04972(17)$ & $0.0380(3)$ \\
\hline $\mathrm{H} 10 \mathrm{~A}$ & 0.3831 & 0.2280 & 1.1351 & $0.046^{*}$ \\
\hline $\mathrm{C} 11$ & $0.5592(2)$ & $0.12282(19)$ & $1.1036(2)$ & $0.0519(4)$ \\
\hline H11A & 0.6423 & 0.1759 & 1.1538 & $0.078^{*}$ \\
\hline H11B & 0.5851 & 0.0865 & 1.0204 & $0.078^{*}$ \\
\hline $\mathrm{H} 11 \mathrm{C}$ & 0.5462 & 0.0616 & 1.1714 & $0.078^{*}$ \\
\hline $\mathrm{C} 12$ & $0.28088(17)$ & 0.10794 (14) & $0.97174(15)$ & $0.0343(3)$ \\
\hline $\mathrm{C} 13$ & 0.04849 (19) & $-0.00018(18)$ & $0.99122(18)$ & $0.0442(4)$ \\
\hline $\mathrm{C} 14$ & $-0.0612(2)$ & $0.0436(2)$ & $0.8479(2)$ & $0.0677(6)$ \\
\hline $\mathrm{H} 14 \mathrm{~A}$ & -0.0073 & 0.0448 & 0.7718 & $0.102 *$ \\
\hline H14B & -0.0963 & 0.1231 & 0.8618 & $0.102 *$ \\
\hline $\mathrm{H} 14 \mathrm{C}$ & -0.1503 & -0.0090 & 0.8183 & $0.102 *$ \\
\hline $\mathrm{C} 15$ & $0.1083(3)$ & $-0.1214(2)$ & $0.9736(3)$ & $0.0666(6)$ \\
\hline $\mathrm{H} 15 \mathrm{~A}$ & 0.1785 & -0.1470 & 1.0665 & $0.100^{*}$ \\
\hline H15B & 0.1635 & -0.1196 & 0.8985 & $0.100^{*}$ \\
\hline $\mathrm{H} 15 \mathrm{C}$ & 0.0216 & -0.1762 & 0.9442 & $0.100^{*}$ \\
\hline C16 & $-0.0360(3)$ & $-0.0012(3)$ & $1.1101(3)$ & $0.0796(8)$ \\
\hline H16B & -0.1251 & -0.0539 & 1.0810 & $0.119^{*}$ \\
\hline $\mathrm{H} 16 \mathrm{C}$ & -0.0710 & 0.0783 & 1.1234 & $0.119^{*}$ \\
\hline H16A & 0.0341 & -0.0289 & 1.2020 & $0.119^{*}$ \\
\hline
\end{tabular}


Atomic displacement parameters $\left(\AA^{2}\right)$

\begin{tabular}{lllllll}
\hline & $U^{11}$ & $U^{22}$ & $U^{33}$ & $U^{12}$ & $U^{13}$ & $U^{23}$ \\
\hline $\mathrm{N} 1$ & $0.0423(7)$ & $0.0407(7)$ & $0.0305(5)$ & $0.0064(6)$ & $0.0173(5)$ & $0.0040(5)$ \\
$\mathrm{N} 2$ & $0.0331(6)$ & $0.0460(8)$ & $0.0282(5)$ & $-0.0053(5)$ & $0.0099(4)$ & $-0.0068(5)$ \\
$\mathrm{O} 1$ & $0.0445(5)$ & $0.0478(7)$ & $0.0295(5)$ & $0.0060(5)$ & $0.0159(4)$ & $0.0053(5)$ \\
$\mathrm{O} 2$ & $0.0592(7)$ & $0.0643(8)$ & $0.0309(5)$ & $-0.0154(6)$ & $0.0208(5)$ & $-0.0115(5)$ \\
$\mathrm{C} 1$ & $0.0598(11)$ & $0.0418(10)$ & $0.0689(12)$ & $-0.0012(8)$ & $0.0318(9)$ & $-0.0113(9)$ \\
$\mathrm{C} 2$ & $0.0438(8)$ & $0.0397(9)$ & $0.0361(7)$ & $0.0048(7)$ & $0.0192(6)$ & $0.0048(6)$ \\
$\mathrm{C} 3$ & $0.0375(9)$ & $0.0540(11)$ & $0.0866(14)$ & $0.0021(8)$ & $0.0229(9)$ & $-0.0120(10)$ \\
$\mathrm{C} 4$ & $0.0373(7)$ & $0.0372(8)$ & $0.0306(6)$ & $-0.0009(6)$ & $0.0135(5)$ & $-0.0003(6)$ \\
$\mathrm{C} 5$ & $0.0390(7)$ & $0.0344(8)$ & $0.0327(7)$ & $0.0047(6)$ & $0.0115(6)$ & $0.0030(6)$ \\
$\mathrm{C} 6$ & $0.0389(8)$ & $0.0390(9)$ & $0.0580(9)$ & $0.0021(7)$ & $0.0168(7)$ & $0.0003(8)$ \\
$\mathrm{C} 7$ & $0.0493(9)$ & $0.0380(8)$ & $0.0429(8)$ & $0.0040(7)$ & $0.0158(7)$ & $-0.0035(7)$ \\
$\mathrm{C} 8$ & $0.0488(9)$ & $0.0495(10)$ & $0.0419(8)$ & $0.0126(8)$ & $0.0134(6)$ & $0.0090(8)$ \\
$\mathrm{C} 9$ & $0.0492(10)$ & $0.0450(10)$ & $0.0796(13)$ & $-0.0033(8)$ & $0.0175(9)$ & $0.0128(10)$ \\
C10 & $0.0388(7)$ & $0.0406(8)$ & $0.0378(7)$ & $-0.0043(7)$ & $0.0159(6)$ & $-0.0073(6)$ \\
C11 & $0.0429(9)$ & $0.0549(11)$ & $0.0519(9)$ & $-0.0034(8)$ & $0.0031(7)$ & $-0.0001(8)$ \\
C12 & $0.0379(7)$ & $0.0401(8)$ & $0.0263(6)$ & $0.0010(6)$ & $0.0111(5)$ & $0.0001(6)$ \\
C13 & $0.0363(7)$ & $0.0559(10)$ & $0.0412(8)$ & $-0.0106(7)$ & $0.0120(6)$ & $-0.0078(8)$ \\
C14 & $0.0457(10)$ & $0.0811(15)$ & $0.0627(12)$ & $-0.0095(10)$ & $-0.0075(8)$ & $-0.0083(11)$ \\
C15 & $0.0647(13)$ & $0.0491(11)$ & $0.0838(15)$ & $-0.0171(10)$ & $0.0172(10)$ & $-0.0049(10)$ \\
C16 & $0.0562(12)$ & $0.120(2)$ & $0.0741(13)$ & $-0.0411(14)$ & $0.0373(10)$ & $-0.0240(15)$ \\
& & & & & &
\end{tabular}

Geometric parameters $\left(A,{ }^{\circ}\right)$

\begin{tabular}{llll}
\hline $\mathrm{N} 1-\mathrm{C} 4$ & $1.331(2)$ & $\mathrm{C} 7-\mathrm{H} 7 \mathrm{C}$ & 0.9800 \\
$\mathrm{~N} 1-\mathrm{C} 5$ & $1.4739(19)$ & $\mathrm{C} 8-\mathrm{H} 8 \mathrm{~A}$ & 0.9800 \\
$\mathrm{~N} 1-\mathrm{H} 1 \mathrm{~A}$ & 0.8800 & $\mathrm{C} 8-\mathrm{H} 8 \mathrm{~B}$ & 0.9800 \\
$\mathrm{~N} 2-\mathrm{C} 12$ & $1.3312(19)$ & $\mathrm{C} 8-\mathrm{H} 8 \mathrm{C}$ & 0.9800 \\
$\mathrm{~N} 2-\mathrm{C} 13$ & $1.475(2)$ & $\mathrm{C} 9-\mathrm{C} 10$ & $1.520(3)$ \\
$\mathrm{N} 2-\mathrm{H} 2 \mathrm{~A}$ & 0.8800 & $\mathrm{C} 9-\mathrm{H} 9 \mathrm{~A}$ & 0.9800 \\
$\mathrm{O} 1-\mathrm{C} 4$ & $1.2328(18)$ & $\mathrm{C} 9-\mathrm{H} 9 \mathrm{~B}$ & 0.9800 \\
$\mathrm{O} 2-\mathrm{C} 12$ & $1.2293(19)$ & $\mathrm{C} 9-\mathrm{H} 9 \mathrm{C}$ & 0.9800 \\
$\mathrm{C} 1-\mathrm{C} 2$ & $1.516(2)$ & $\mathrm{C} 10-\mathrm{C} 11$ & $1.523(2)$ \\
$\mathrm{C} 1-\mathrm{H} 1 \mathrm{~B}$ & 0.9800 & $\mathrm{C} 10-\mathrm{C} 12$ & $1.531(2)$ \\
$\mathrm{C} 1-\mathrm{H} 1 \mathrm{C}$ & 0.9800 & $\mathrm{C} 10-\mathrm{H} 10 \mathrm{~A}$ & 1.0000 \\
$\mathrm{C} 1-\mathrm{H} 1 \mathrm{D}$ & 0.9800 & $\mathrm{C} 11-\mathrm{H} 11 \mathrm{~A}$ & 0.9800 \\
$\mathrm{C} 2-\mathrm{C} 3$ & $1.519(2)$ & $\mathrm{C} 11-\mathrm{H} 11 \mathrm{~B}$ & 0.9800 \\
$\mathrm{C} 2-\mathrm{C} 4$ & $1.544(2)$ & $\mathrm{C} 11-\mathrm{H} 11 \mathrm{C}$ & 0.9800 \\
$\mathrm{C} 2-\mathrm{H} 2 \mathrm{~B}$ & 1.0000 & $\mathrm{C} 13-\mathrm{C} 15$ & $1.509(3)$ \\
$\mathrm{C} 3-\mathrm{H} 3 \mathrm{~A}$ & 0.9800 & $\mathrm{C} 13-\mathrm{C} 14$ & $1.533(3)$ \\
$\mathrm{C} 3-\mathrm{H} 3 \mathrm{~B}$ & 0.9800 & $\mathrm{C} 13-\mathrm{C} 16$ & $1.533(3)$ \\
$\mathrm{C} 3-\mathrm{H} 3 \mathrm{C}$ & 0.9800 & $\mathrm{C} 14-\mathrm{H} 14 \mathrm{~A}$ & 0.9800 \\
$\mathrm{C} 5-\mathrm{C} 7$ & $1.523(2)$ & $\mathrm{C} 14-\mathrm{H} 14 \mathrm{~B}$ & 0.9800 \\
$\mathrm{C} 5-\mathrm{C} 6$ & $1.527(2)$ & $\mathrm{C} 14-\mathrm{H} 14 \mathrm{C}$ & 0.9800 \\
$\mathrm{C} 5-\mathrm{C} 8$ & $1.530(2)$ & $\mathrm{C} 15-\mathrm{H} 15 \mathrm{~A}$ & 0.9800 \\
$\mathrm{C} 6-\mathrm{H} 6 \mathrm{~A}$ & 0.9800 & $\mathrm{C} 15-\mathrm{H} 15 \mathrm{~B}$ & 0.9800
\end{tabular}




\begin{tabular}{|c|c|c|c|}
\hline $\mathrm{C} 6-\mathrm{H} 6 \mathrm{~B}$ & 0.9800 & $\mathrm{C} 15-\mathrm{H} 15 \mathrm{C}$ & 0.9800 \\
\hline $\mathrm{C} 6-\mathrm{H} 6 \mathrm{C}$ & 0.9800 & $\mathrm{C} 16-\mathrm{H} 16 \mathrm{~B}$ & 0.9800 \\
\hline $\mathrm{C} 7-\mathrm{H} 7 \mathrm{~A}$ & 0.9800 & $\mathrm{C} 16-\mathrm{H} 16 \mathrm{C}$ & 0.9800 \\
\hline C7-H7B & 0.9800 & $\mathrm{C} 16-\mathrm{H} 16 \mathrm{~A}$ & 0.9800 \\
\hline $\mathrm{C} 4-\mathrm{N} 1-\mathrm{C} 5$ & $126.13(11)$ & $\mathrm{C} 5-\mathrm{C} 8-\mathrm{H} 8 \mathrm{C}$ & 109.5 \\
\hline $\mathrm{C} 4-\mathrm{N} 1-\mathrm{H} 1 \mathrm{~A}$ & 116.9 & $\mathrm{H} 8 \mathrm{~A}-\mathrm{C} 8-\mathrm{H} 8 \mathrm{C}$ & 109.5 \\
\hline $\mathrm{C} 5-\mathrm{N} 1-\mathrm{H} 1 \mathrm{~A}$ & 116.9 & $\mathrm{H} 8 \mathrm{~B}-\mathrm{C} 8-\mathrm{H} 8 \mathrm{C}$ & 109.5 \\
\hline $\mathrm{C} 12-\mathrm{N} 2-\mathrm{C} 13$ & $124.63(13)$ & $\mathrm{C} 10-\mathrm{C} 9-\mathrm{H} 9 \mathrm{~A}$ & 109.5 \\
\hline $\mathrm{C} 12-\mathrm{N} 2-\mathrm{H} 2 \mathrm{~A}$ & 117.7 & $\mathrm{C} 10-\mathrm{C} 9-\mathrm{H} 9 \mathrm{~B}$ & 109.5 \\
\hline $\mathrm{C} 13-\mathrm{N} 2-\mathrm{H} 2 \mathrm{~A}$ & 117.7 & $\mathrm{H} 9 \mathrm{~A}-\mathrm{C} 9-\mathrm{H} 9 \mathrm{~B}$ & 109.5 \\
\hline $\mathrm{C} 2-\mathrm{C} 1-\mathrm{H} 1 \mathrm{~B}$ & 109.5 & $\mathrm{C} 10-\mathrm{C} 9-\mathrm{H} 9 \mathrm{C}$ & 109.5 \\
\hline $\mathrm{C} 2-\mathrm{C} 1-\mathrm{H} 1 \mathrm{C}$ & 109.5 & $\mathrm{H} 9 \mathrm{~A}-\mathrm{C} 9-\mathrm{H} 9 \mathrm{C}$ & 109.5 \\
\hline $\mathrm{H} 1 \mathrm{~B}-\mathrm{C} 1-\mathrm{H} 1 \mathrm{C}$ & 109.5 & $\mathrm{H} 9 \mathrm{~B}-\mathrm{C} 9-\mathrm{H} 9 \mathrm{C}$ & 109.5 \\
\hline $\mathrm{C} 2-\mathrm{C} 1-\mathrm{H} 1 \mathrm{D}$ & 109.5 & $\mathrm{C} 9-\mathrm{C} 10-\mathrm{C} 11$ & $110.23(15)$ \\
\hline $\mathrm{H} 1 \mathrm{~B}-\mathrm{C} 1-\mathrm{H} 1 \mathrm{D}$ & 109.5 & $\mathrm{C} 9-\mathrm{C} 10-\mathrm{C} 12$ & $109.85(13)$ \\
\hline $\mathrm{H} 1 \mathrm{C}-\mathrm{C} 1-\mathrm{H} 1 \mathrm{D}$ & 109.5 & $\mathrm{C} 11-\mathrm{C} 10-\mathrm{C} 12$ & $108.96(14)$ \\
\hline $\mathrm{C} 1-\mathrm{C} 2-\mathrm{C} 3$ & $111.37(16)$ & $\mathrm{C} 9-\mathrm{C} 10-\mathrm{H} 10 \mathrm{~A}$ & 109.3 \\
\hline $\mathrm{C} 1-\mathrm{C} 2-\mathrm{C} 4$ & $109.28(14)$ & $\mathrm{C} 11-\mathrm{C} 10-\mathrm{H} 10 \mathrm{~A}$ & 109.3 \\
\hline $\mathrm{C} 3-\mathrm{C} 2-\mathrm{C} 4$ & $109.74(14)$ & $\mathrm{C} 12-\mathrm{C} 10-\mathrm{H} 10 \mathrm{~A}$ & 109.3 \\
\hline $\mathrm{C} 1-\mathrm{C} 2-\mathrm{H} 2 \mathrm{~B}$ & 108.8 & $\mathrm{C} 10-\mathrm{C} 11-\mathrm{H} 11 \mathrm{~A}$ & 109.5 \\
\hline $\mathrm{C} 3-\mathrm{C} 2-\mathrm{H} 2 \mathrm{~B}$ & 108.8 & $\mathrm{C} 10-\mathrm{C} 11-\mathrm{H} 11 \mathrm{~B}$ & 109.5 \\
\hline $\mathrm{C} 4-\mathrm{C} 2-\mathrm{H} 2 \mathrm{~B}$ & 108.8 & $\mathrm{H} 11 \mathrm{~A}-\mathrm{C} 11-\mathrm{H} 11 \mathrm{~B}$ & 109.5 \\
\hline $\mathrm{C} 2-\mathrm{C} 3-\mathrm{H} 3 \mathrm{~A}$ & 109.5 & $\mathrm{C} 10-\mathrm{C} 11-\mathrm{H} 11 \mathrm{C}$ & 109.5 \\
\hline $\mathrm{C} 2-\mathrm{C} 3-\mathrm{H} 3 \mathrm{~B}$ & 109.5 & $\mathrm{H} 11 \mathrm{~A}-\mathrm{C} 11-\mathrm{H} 11 \mathrm{C}$ & 109.5 \\
\hline $\mathrm{H} 3 \mathrm{~A}-\mathrm{C} 3-\mathrm{H} 3 \mathrm{~B}$ & 109.5 & $\mathrm{H} 11 \mathrm{~B}-\mathrm{C} 11-\mathrm{H} 11 \mathrm{C}$ & 109.5 \\
\hline $\mathrm{C} 2-\mathrm{C} 3-\mathrm{H} 3 \mathrm{C}$ & 109.5 & $\mathrm{O} 2-\mathrm{C} 12-\mathrm{N} 2$ & $123.37(15)$ \\
\hline $\mathrm{H} 3 \mathrm{~A}-\mathrm{C} 3-\mathrm{H} 3 \mathrm{C}$ & 109.5 & $\mathrm{O} 2-\mathrm{C} 12-\mathrm{C} 10$ & $120.91(14)$ \\
\hline $\mathrm{H} 3 \mathrm{~B}-\mathrm{C} 3-\mathrm{H} 3 \mathrm{C}$ & 109.5 & $\mathrm{~N} 2-\mathrm{C} 12-\mathrm{C} 10$ & $115.72(13)$ \\
\hline $\mathrm{O} 1-\mathrm{C} 4-\mathrm{N} 1$ & $124.59(14)$ & $\mathrm{N} 2-\mathrm{C} 13-\mathrm{C} 15$ & $110.67(15)$ \\
\hline $\mathrm{O} 1-\mathrm{C} 4-\mathrm{C} 2$ & $120.20(14)$ & $\mathrm{N} 2-\mathrm{C} 13-\mathrm{C} 14$ & $110.00(16)$ \\
\hline $\mathrm{N} 1-\mathrm{C} 4-\mathrm{C} 2$ & $115.22(12)$ & $\mathrm{C} 15-\mathrm{C} 13-\mathrm{C} 14$ & $111.19(18)$ \\
\hline $\mathrm{N} 1-\mathrm{C} 5-\mathrm{C} 7$ & $111.09(12)$ & $\mathrm{N} 2-\mathrm{C} 13-\mathrm{C} 16$ & $105.46(15)$ \\
\hline $\mathrm{N} 1-\mathrm{C} 5-\mathrm{C} 6$ & $109.64(12)$ & $\mathrm{C} 15-\mathrm{C} 13-\mathrm{C} 16$ & $110.0(2)$ \\
\hline $\mathrm{C} 7-\mathrm{C} 5-\mathrm{C} 6$ & $111.15(14)$ & $\mathrm{C} 14-\mathrm{C} 13-\mathrm{C} 16$ & $109.32(18)$ \\
\hline $\mathrm{N} 1-\mathrm{C} 5-\mathrm{C} 8$ & $106.60(12)$ & $\mathrm{C} 13-\mathrm{C} 14-\mathrm{H} 14 \mathrm{~A}$ & 109.5 \\
\hline $\mathrm{C} 7-\mathrm{C} 5-\mathrm{C} 8$ & $108.50(14)$ & $\mathrm{C} 13-\mathrm{C} 14-\mathrm{H} 14 \mathrm{~B}$ & 109.5 \\
\hline $\mathrm{C} 6-\mathrm{C} 5-\mathrm{C} 8$ & $109.75(13)$ & $\mathrm{H} 14 \mathrm{~A}-\mathrm{C} 14-\mathrm{H} 14 \mathrm{~B}$ & 109.5 \\
\hline $\mathrm{C} 5-\mathrm{C} 6-\mathrm{H} 6 \mathrm{~A}$ & 109.5 & $\mathrm{C} 13-\mathrm{C} 14-\mathrm{H} 14 \mathrm{C}$ & 109.5 \\
\hline $\mathrm{C} 5-\mathrm{C} 6-\mathrm{H} 6 \mathrm{~B}$ & 109.5 & $\mathrm{H} 14 \mathrm{~A}-\mathrm{C} 14-\mathrm{H} 14 \mathrm{C}$ & 109.5 \\
\hline $\mathrm{H} 6 \mathrm{~A}-\mathrm{C} 6-\mathrm{H} 6 \mathrm{~B}$ & 109.5 & $\mathrm{H} 14 \mathrm{~B}-\mathrm{C} 14-\mathrm{H} 14 \mathrm{C}$ & 109.5 \\
\hline $\mathrm{C} 5-\mathrm{C} 6-\mathrm{H} 6 \mathrm{C}$ & 109.5 & $\mathrm{C} 13-\mathrm{C} 15-\mathrm{H} 15 \mathrm{~A}$ & 109.5 \\
\hline $\mathrm{H} 6 \mathrm{~A}-\mathrm{C} 6-\mathrm{H} 6 \mathrm{C}$ & 109.5 & $\mathrm{C} 13-\mathrm{C} 15-\mathrm{H} 15 \mathrm{~B}$ & 109.5 \\
\hline $\mathrm{H} 6 \mathrm{~B}-\mathrm{C} 6-\mathrm{H} 6 \mathrm{C}$ & 109.5 & $\mathrm{H} 15 \mathrm{~A}-\mathrm{C} 15-\mathrm{H} 15 \mathrm{~B}$ & 109.5 \\
\hline $\mathrm{C} 5-\mathrm{C} 7-\mathrm{H} 7 \mathrm{~A}$ & 109.5 & $\mathrm{C} 13-\mathrm{C} 15-\mathrm{H} 15 \mathrm{C}$ & 109.5 \\
\hline $\mathrm{C} 5-\mathrm{C} 7-\mathrm{H} 7 \mathrm{~B}$ & 109.5 & $\mathrm{H} 15 \mathrm{~A}-\mathrm{C} 15-\mathrm{H} 15 \mathrm{C}$ & 109.5 \\
\hline $\mathrm{H} 7 \mathrm{~A}-\mathrm{C} 7-\mathrm{H} 7 \mathrm{~B}$ & 109.5 & $\mathrm{H} 15 \mathrm{~B}-\mathrm{C} 15-\mathrm{H} 15 \mathrm{C}$ & 109.5 \\
\hline $\mathrm{C} 5-\mathrm{C} 7-\mathrm{H} 7 \mathrm{C}$ & 109.5 & $\mathrm{C} 13-\mathrm{C} 16-\mathrm{H} 16 \mathrm{~B}$ & 109.5 \\
\hline
\end{tabular}




\begin{tabular}{llll}
$\mathrm{H} 7 \mathrm{~A}-\mathrm{C} 7-\mathrm{H} 7 \mathrm{C}$ & 109.5 & $\mathrm{C} 13-\mathrm{C} 16-\mathrm{H} 16 \mathrm{C}$ & 109.5 \\
$\mathrm{H} 7 \mathrm{~B}-\mathrm{C} 7-\mathrm{H} 7 \mathrm{C}$ & 109.5 & $\mathrm{H} 16 \mathrm{~B}-\mathrm{C} 16-\mathrm{H} 16 \mathrm{C}$ & 109.5 \\
$\mathrm{C} 5-\mathrm{C} 8-\mathrm{H} 8 \mathrm{~A}$ & 109.5 & $\mathrm{C} 13-\mathrm{C} 16-\mathrm{H} 16 \mathrm{~A}$ & 109.5 \\
$\mathrm{C} 5-\mathrm{C} 8-\mathrm{H} 8 \mathrm{~B}$ & 109.5 & $\mathrm{H} 16 \mathrm{~B}-\mathrm{C} 16-\mathrm{H} 16 \mathrm{~A}$ & 109.5 \\
$\mathrm{H} 8 \mathrm{~A}-\mathrm{C} 8-\mathrm{H} 8 \mathrm{~B}$ & 109.5 & $\mathrm{H} 16 \mathrm{C}-\mathrm{C} 16-\mathrm{H} 16 \mathrm{~A}$ & 109.5 \\
& & & \\
$\mathrm{C} 5-\mathrm{N} 1-\mathrm{C} 4-\mathrm{O} 1$ & $3.9(3)$ & $\mathrm{C} 13-\mathrm{N} 2-\mathrm{C} 12-\mathrm{O} 2$ & $2.5(3)$ \\
$\mathrm{C} 5-\mathrm{N} 1-\mathrm{C} 4-\mathrm{C} 2$ & $-175.58(14)$ & $\mathrm{C} 13-\mathrm{N} 2-\mathrm{C} 12-\mathrm{C} 10$ & $-176.63(15)$ \\
$\mathrm{C} 1-\mathrm{C} 2-\mathrm{C} 4-\mathrm{O} 1$ & $-63.0(2)$ & $\mathrm{C} 9-\mathrm{C} 10-\mathrm{C} 12-\mathrm{O} 2$ & $49.6(2)$ \\
$\mathrm{C} 3-\mathrm{C} 2-\mathrm{C} 4-\mathrm{O} 1$ & $59.4(2)$ & $\mathrm{C} 11-\mathrm{C} 10-\mathrm{C} 12-\mathrm{O} 2$ & $-71.2(2)$ \\
$\mathrm{C} 1-\mathrm{C} 2-\mathrm{C} 4-\mathrm{N} 1$ & $116.50(17)$ & $\mathrm{C} 9-\mathrm{C} 10-\mathrm{C} 12-\mathrm{N} 2$ & $-131.26(16)$ \\
$\mathrm{C} 3-\mathrm{C} 2-\mathrm{C} 4-\mathrm{N} 1$ & $-121.10(17)$ & $\mathrm{C} 11-\mathrm{C} 10-\mathrm{C} 12-\mathrm{N} 2$ & $107.89(17)$ \\
$\mathrm{C} 4-\mathrm{N} 1-\mathrm{C} 5-\mathrm{C} 7$ & $-60.1(2)$ & $\mathrm{C} 12-\mathrm{N} 2-\mathrm{C} 13-\mathrm{C} 15$ & $59.7(2)$ \\
$\mathrm{C} 4-\mathrm{N} 1-\mathrm{C} 5-\mathrm{C} 6$ & $63.11(19)$ & $\mathrm{C} 12-\mathrm{N} 2-\mathrm{C} 13-\mathrm{C} 14$ & $-63.5(2)$ \\
$\mathrm{C} 4-\mathrm{N} 1-\mathrm{C} 5-\mathrm{C} 8$ & $-178.15(16)$ & $\mathrm{C} 12-\mathrm{N} 2-\mathrm{C} 13-\mathrm{C} 16$ & $178.7(2)$ \\
\hline
\end{tabular}

Hydrogen-bond geometry $\left(A,{ }^{\circ}\right)$

\begin{tabular}{lllll}
\hline$D-\mathrm{H} \cdots A$ & $D-\mathrm{H}$ & $\mathrm{H} \cdots A$ & $D \cdots A$ & $D-\mathrm{H} \cdots A$ \\
\hline $\mathrm{N} 1-\mathrm{H} 1 A \cdots \mathrm{O} 2$ & 0.88 & 2.03 & $2.8880(16)$ & 166 \\
$\mathrm{~N} 2-\mathrm{H} 2 A \cdots \mathrm{O} 1^{\mathrm{i}}$ & 0.88 & 2.10 & $2.9735(16)$ & 169 \\
\hline
\end{tabular}

Symmetry code: (i) $x, y, z+1$. 